\title{
Comprendre les luttes sociales dans et depuis le Sud Global
}

\author{
Entretien avec Raquel Gutiérrez Aguilar
}

\begin{abstract}
Raquel Gutiérrez Aguilar, Elisabeth Lagasse ${ }^{1}$

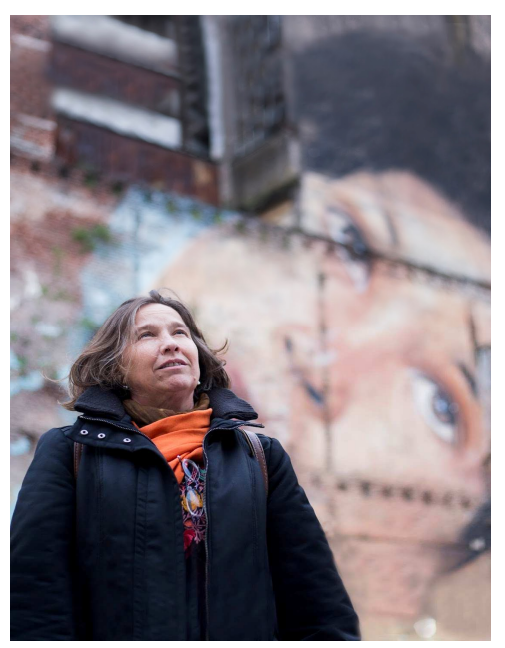

Cet entretien a été réalisé en espagnol le 22 novembre 2016 lors d'un séjour de recherche à l'Institut Alfonso Vélez Pliego et traduit par Elisabeth Lagasse, avec l'aide de Raúl Lara et Margot Achard.

Raquel Gutiérrez Aguilar est professeure de sociologie et chercheuse à l'Instituto de Ciencias Sociales y Humanidades Alfonso Vélez Pliego de la Benemérita Universidad Autónoma de Puebla, au Mexique. Mathématicienne, philosophe et sociologue, elle a milité dans les années 1990 en Bolivie au sein de l'Ejército Guerrillero Tupac Katari (EGTK), une organisation principalement Aymara. Son engagement lui a valu d'être emprisonnée plusieurs années. Ses thèmes de recherche sont les mouvements indigènes et populaires, en particulier en Bolivie, les mouvements féministes, la politique du commun. En 1999, elle a publié Desandar el laberinto. Introspección en la feminidad contemporánea aux éditions Paz en el arbol. Elle est également l'auteure de $;$ A desordenar ! Por una historia abierta de la lucha social (2006), publié aux éditions Casa Juan Pablos, ainsi que de Los ritmos del Pachakuti : movilización y levantamiento popular-indígena en Bolivia (2000-2005) (2009) chez Tierra Ediciones (disponible en anglais aux éditions Duke University Press [2014]).
\end{abstract}

Pour commencer, pouvez-vous nous parler de votre parcours intellectuel ? Comment vos parcours intellectuels et militants se sont-ils nourris mutuellement?

RGA : Je viens d'une tradition scientifique un peu insolite parce que j'ai étudié les mathématiques en licence, il y a très longtemps, dans les années 1980, lorsque la carrière

1 Centre de recherches interdisciplinaires Démocratie, Institutions, Subjectivité (CRIDIS). 
académique n'était pas nécessairement une option privilégiée par les jeunes. Même si beaucoup de personnes faisaient des carrières académiques, ce n'était pas l'horizon, pour le dire ainsi. On était aussi dans un monde totalement différent de celui qui existe aujourd'hui : un monde où il y avait du travail, un peu plus de sécurité. J'aimerais souligner un problème important pour les camarades de ta génération : quand j'étudiais les mathématiques à l'UNAM, pour pouvoir survivre sans bourse, je devais travailler deux heures par jour. C'est-à-dire qu'en tant qu'étudiante régulière d'une université publique, en donnant deux heures de cours de mathématiques à des adolescents, j'avais suffisamment de ressources pour financer ma propre vie, autrement dit payer mon loyer, acheter mes livres, ma nourriture, et aller en vacances. Donc, ce monde qui donnait tant de sécurité entraînait aussi une autre forme de subjectivité, parce qu'on ne devait pas systématiquement chercher à financer les choses. Ma génération est la dernière qui a eu ces opportunités. J'ai donc étudié les mathématiques et, au début des années 1980, j'ai initié une trajectoire militante au sein du vieux groupe de gauche classique, alors qu'un ensemble de certitudes du XX $\mathrm{XX}^{\mathrm{e}}$ siècle étaient déjà en train d'entrer en crise. Ce fut ma formation militante initiale : je me suis formée intellectuellement par le marxisme, le léninisme canonique, je me suis nourrie des discussions sur la guerre en Amérique centrale, d'auteurs d'Afrique du Nord, de Fanon, des discussions sur les luttes anticoloniales, etc. J'ai appris deux choses : d'un côté, des modes de raisonnement et d'argumentation en étudiant les mathématiques, et de l'autre, j'ai eu une formation conforme à celle d'une militante de gauche radicale de la décennie des années 1980.

Ensuite, il y a eu un autre élément qui s'est très vite imbriqué dans cette trajectoire intellectuelle, vers le milieu des années 1980, quand je vivais en Bolivie. Il s'agissait d'apprendre à essayer de comprendre, de façon très indirecte, très profonde, un ensemble de pratiques et de discours qui permettaient de saisir une certaine cosmovision indigène de l'Altiplano bolivien. J’ai en effet vécu et partagé la vie quotidienne, pendant de nombreuses années, avec des personnes issues du mouvement aymara et j'étais en relation permanente avec beaucoup d'entre eux. Une série d'études anthropologiques dans toute l'aire andine ont été réalisées à cette époque, justement par des chercheurs principalement francophones. Apprécier la façon dont ces études traitaient cette question a aussi été très important dans ma formation. Mais surtout, j'ai eu la possibilité de mener une réflexion quotidienne avec cet ensemble d'hommes et de femmes issus de différentes communautés principalement aymaras et ensuite quechuas. J'ai eu l'occasion de dialoguer avec eux, avec un statut d'égal à égal, sur tout ce que je savais de ma formation de militante marxiste-léniniste.

Ce sont donc les trois sources qui m'ont formée et qui constituent ma façon de penser. Un élément supplémentaire à cette formation intellectuelle, c'était ma qualité de femme inscrite dans des organisations de gauche de domination masculine, pour ne pas dire directement de forme patriarcale. J'ai été par la suite de plus en plus exposée aux luttes déployées par les femmes organisées de la gauche, de la fin des années 1970 et du début des années 1980. En effet, bien que sans discours féministe formel, elles 
menaient un combat très clair, en particulier pour le respect à l'organisation spécifique des femmes et leur autonomie dans les luttes mixtes. Ainsi, ce sont mes quatre sources : ma formation académique d'une certaine rigueur; un marxisme-léninisme étudié étape par étape, comme il s'étudiait à cette époque-là ; une vie quotidienne auprès du mouvement aymara en développement et le fait d'être moi-même femme en lutte. C'est ce qui m’a constitué.

Ressentez-vous une tension entre les conditions de militante et de chercheuse? Quelles sont les difficultés qui en découlent?

RGA : Je n'en vois plus maintenant, parce qu'en plus ce sont deux vies, deux moments distincts de ma vie. J'étais une militante qui, en même temps, réfléchissait, pensait, développait un discours sur le plan intellectuel. C'est ce que j'ai fait avec un ensemble d'autres personnes, en stimulant une discussion dans le sillon du marxisme, dans le sillon communautaire et dans l'indianisme, et jusqu'à un certain point, dans le sillon féministe. Mais être chercheuse, c'est très différent d'être militante, parce que je n'étais pas une activiste, mais une militante, et une militante organisée dans une structure, qui produit des documents et des arguments de densité différente. Les uns plus pour la propagande, les autres plus pour la dispute du sens, d'autres, encore plus théoriques, dans une perspective d'organisation. Et ce, de façon complètement autonome et ardue, parce que c'était du temps soutiré à d'autres tâches. Mais en même temps, il fallait être bien attentif aux conséquences de ses mots, c'est-à-dire, savoir qu'on est en train de construire des arguments, parce qu'on veut qu'ils aient une efficacité immédiate, dans le domaine de l'intervention politique. Cela a été la première partie de mon expérience.

Par la suite, et pour un ensemble de raisons, je suis retournée à Mexico au début des années 2000 après avoir vécu en Bolivie pendant 17 ans. Dès mon retour, j’ai commencé à combiner une étude universitaire plus rigoureuse avec une tentative d'intervention politique allant dans le sens de la critique de discussions fondamentales portant sur le sens même du politique. Mais du coup, je commençais à changer, à devenir une intellectuelle qui intervient politiquement. Ce sont donc deux expériences très différentes. Et oui, je vois une complication dans cette seconde expérience. En fait, si je fais un bilan de ma vie, je ressens que j'étais plus à l'aise dans le rôle de militante, qui était plus fluide. Aujourd'hui, dans cette autre expérience, étant ce que je suis, c'est-àdire une professeure titulaire de troisième cycle, qui a beaucoup d'étudiants, qui travaille dans un domaine de recherche, qui a une certaine autorité, je sens que ça me demande beaucoup plus d'efforts de parvenir à la production d'idées qui peuvent avoir une efficacité politique rapide. Et parfois, je pense que ce que je dois faire et ce que je suis en train de faire, c'est évoluer vers une position plus stratégique, à plus long terme.

Pour terminer et finir sur cette question, j'ai mené plusieurs projets d'enquête de terrain durant presque six années et je vais bientôt commencer un nouveau travail de 
terrain, dans lequel je vais recommencer à essayer, comme dans ma jeunesse, de combiner ces deux postures. Parce que je veux approfondir et mettre davantage l'accent sur la dérive féministe dans ma réflexion. D'autant plus que je remarque une sorte de moment d'ébullition, d'énergies qui sont en train de jaillir, que je salue, auquel je participe et dont je suis très heureuse. Et je veux faire partie de ça, et aussi réfléchir à ce sujet à cette nouvelle étape de ma vie, où j'essaye de regrouper en une forme plus fluide, mes deux expériences antérieures.

\section{Comment le féminisme a-t-il influencé votre travail et perspective de recherche?}

RGA : J'ai commencé à penser la question du féminisme à deux moments : dans la foulée des luttes des femmes, au milieu des grandes luttes déployées depuis la gauche armée et non-armée des années 1970 et 1980 ; puis, avec quelques camarades, lorsque nous avions une revue qui portait le nom de Féminisme prolétaire (qui ne pouvait s'appeler autrement à cette époque-là, n'est-ce pas), et qui diagnostiquait sous certaines formes l'objectif des discussions sur le féminisme. Si je fais le bilan de tout cela, je crois que pendant très longtemps, le féminisme n'était pas un thème tant central dans mes recherches, mais bien de ma vie. Et donc, j'ai tissé des liens avec beaucoup de femmes, camarades, qui après se sont consolidés et ont débouché sur la création de la Confédération des femmes paysannes indigènes Bartolina Sisa. Cette dernière a beaucoup travaillé pour essayer d'établir des relations plus ou moins équilibrées avec les hommes... Mais, la composante féministe explicite et directe était un peu éteinte.

Ma réflexion sur le féminisme s'est relancée durant mon emprisonnement et en sortant de prison. J'avais alors plus clairement, et plus fortement, conscience de la nécessité de subversion des relations de pouvoir et de hiérarchies qui découlent de l'ordre patriarcal du capital, ce qui est une entreprise très difficile. Au fur et à mesure, c'est devenu beaucoup plus évident pour moi et j'ai commencé à essayer de le réaliser pour moi-même. J'ai ainsi écrit un petit livre en 1999, Desander el laberinto : Introspección en la feminidad contemporánea, qui est une introspection sur ma propre féminité, dans le but de comprendre un ensemble d'éléments que je n'avais pas fixé jusqu'à présent et, en essayant de me lier avec d'autres femmes, plus jeunes que moi, de façon à mieux connaître les situations qu'on ne peut pas accepter.

Donc, disons que mon rapprochement au féminisme a toujours été un peu canonique, parce qu'à la fin de ma période militante, tout d'un coup ce qui était un mouvement féministe très puissant, qui avait de multiples facettes et convergences, se retourna, et se convertit en débats sur le genre, qui d'un côté ont fait leur entrée dans le monde scientifique avec des études sur les femmes, et d'un autre côté entrait dans l'espace public par un agenda sur l'équité et les droits sexuels et reproductifs. Et je ne suis pas au clair avec ce passage. Je recommence donc à y accorder de l'attention, à le penser depuis ma propre féminité avec mon propre regard. Celui-ci va se confronter à deux autres idées très importantes, qui sont d'une part, tout ce que j'ai appris sur la force des 
cadres communautaires de la voie indigène dans laquelle j’ai développé une partie de mon travail intellectuel et politique, et d'autre part l'effort de préciser d'autres choses que j'ai vécues durant cette période et de ces discussions diverses qui m'ont nourrie.

Mais déjà, à la fin des années 1990, j'avais commencé deux choses : d'une part, essayer de contribuer à la mise en place d'un ensemble de groupes de femmes qui se pensent comme groupes de femmes, comme espaces de femmes, où l'on commence de nouveau à récupérer la vieille tradition féministe de l'auto-conscience comme façon de comprendre et de s'armer peu à peu. Mais aussi d'autre part à penser cela en termes politiques, c'est-à-dire à penser les termes de la politisation possible de cette force d'être entre femmes. Et de là, j'ai progressivement essayé d'intégrer à mon travail intellectuel une voie qui se rapproche plus du mouvement indigène, de la force de la vie communautaire, pour perturber les institutions de la démocratie procédurale, pour défier les termes de l'accumulation capitaliste. Je crois que c'est ce qui ouvre un horizon de transformation possible.

J'y ai consacré beaucoup de temps. Durant les premières années de la décennie 2000, nous avons écrit de manière systématique, avec une autre collègue, un ensemble de livres sur le mouvement indigène en Amérique Latine. Ce sont trois volumes qui documentent ce qui se passe dans différents pays, en faisant dialoguer beaucoup d'acteurs, à la fois des dirigeants et des personnes qui participent au mouvement. Au début des années 2000, quand j'étais déjà rentrée au Mexique, la polémique ouverte par le zapatisme portait sur la question de savoir comment déstabiliser l'État pour pouvoir inclure ou incorporer ces droits, et jusqu'où cela pouvait être un chemin de diversion de la forme étatique en soi et pas seulement une forme de contention du mouvement qui au final renforcerait le modèle étatique de la domination. Car il existe en fait toujours une espèce de processus d'amplification, d'inclusion subordonnée. Il y a peut-être un processus de démocratisation partielle de certaines institutions étatiques, mais les côtés plus aiguisés de la contestation mis en jeu dans la lutte elle-même, dans le blocage, dans le soulèvement sont, dans le même, temps dompté, limité, capturé, réprimé. Cela a été un objet de mon attention et j'ai privilégié de façon quasi-exclusive le travail avec les femmes. Autrement dit, j'ai commencé à sentir que je voulais travailler chaque fois plus, en me sentant à l'aise, avec une équipe de camarades femmes avec lesquelles nous pouvions politiser ces éléments pour déranger les relations de pouvoir, de façon à rendre plus fluides les interactions avec certains camarades masculins. Je veux maintenant reprendre réellement la réflexion sur cette dérive féministe avec plus d'insistance, ce à quoi je me consacrerai dans les années qui me restent.

\section{Existe-t-il également une lutte des femmes à l'intérieur de l'université?}

RGA : Oui, c'est un monde très masculin, durement masculin. Il y a un personnage de dessin animé qui s'appelle Nacho Progre et qui représente bien ce que dit un vieux mot d'ordre selon lequel rien ne se rapproche plus d'un machiste de droite qu'un machiste 
de gauche. Je sens que cette université, qui a été consolidée par un ensemble d'hommes de gauche de la vieille garde, s'il y a bien quelque chose qu'elle n'a pas réussi à mettre en doute, sur laquelle elle n'a pas réussi à mener une réflexion de fond, c'est sur le contenu de ses pratiques misogynes, patriarcales, de l'ordre dominant et hiérarchique, ce qui place les hommes aux postes privilégiés et ce que l'Université ne veut pas penser. Et donc, cette figure de Nacho Progre (Progre de progressiste), permet de prendre conscience de quelque chose que nous pourrions appeler le machisme cordial, en faisant un rapprochement avec ce que l'on appelle au Mexique le racisme cordial, c'est-à-dire un racisme qui n'est pas celui de Trump. Ce Nacho Progre, nous met au courant de cette misogynie cordiale, de ce machisme cordial duquel il est imprégné. C'est quelque chose que nous essayons de travailler, et qui a à voir avec ce que je t’ai raconté : récupérer un lieu de plein combat qui va alimenter la pensée, et non l'inverse. Ne pas se placer dans une espèce de lieu qui est un lieu de simple théorie. La carrière académique nous pousse à prendre cette direction, de la même façon que je me suis rangée ici à l'université, pour faire référence à la première partie de la question.

Donc, avec ces luttes féministes qui se manifestent actuellement il s'agit d'essayer de mettre l'énergie dans tout cet ensemble de choses qu'il faut transformer : les petites, les moyennes et les grandes ; les quotidiennes et les structurelles ; les subtiles et les grossières. C'est-à-dire, lutter contre le féminicide, oui, mais aussi lutter contre le monopole de la parole dans une classe par les camarades masculins; lutter pour un droit à un avortement légal et un droit à décider, mais aussi lutter quotidiennement pour établir des termes d'inclusion confortable dans les structures qui doivent se dés-imprégner de ce caractère patriarcal tellement brutal.

Quelle est votre perspective théorique pour étudier ces luttes? Pourquoi choisir de les étudier depuis la théorie critique et le marxisme "ouvert» plutôt que depuis une théorie des mouvements sociaux?

RGA : Je me suis abreuvée de la théorie critique, et quand je suis rentrée au Mexique, j'ai trouvé des interlocuteurs très lucides et ça a été très intéressant pour moi de dialoguer avec la théorie critique. Il y avait alors un effort de rénovation, afin de cultiver une ouverture du vieux marxisme. Aujourd'hui, j'essaye de construire un regard qui ne s'inscrit pas pleinement dans les termes de la théorie critique. Je critique l'idée de mouvements sociaux et sa sociologie, à cause de ce qu'il s'est passé, et surtout suite aux apports d'auteurs anglo-saxons qui ont commencé à être très connus à l'époque de la grande vague de mobilisations et de rébellions dans presque tous les pays d'Amérique Latine, plus ou moins à la fin des années 1990 et au début des années 2000. Ces théories établissaient des codes identificatoires qui ont finalement mené à fermer la dynamique d'ouverture qui se créait à partir des mouvements sociaux. 
Je vais expliquer un peu cette histoire. Le problème central de toutes les discussions $\mathrm{du} \mathrm{XX} \mathrm{X}^{\mathrm{e}}$ siècle était celui du Sujet de la transformation. Ce sera toute la discussion de ce siècle autour du contenu du terme classe ouvrière ou du terme prolétaire. Et le problème avec la théorie des mouvements sociaux est qu'elle ne parvient pas à faire une critique radicale de certains éléments qui structuraient la théorie du grand Sujet historique. Elle a simplement rompu avec le général universel qui établissait le terme classe ouvrière pour le généraliser sous un autre terme. Mais elle a conservé l'ordre argumentatif, et cela a amené en Bolivie, par exemple, à quelque chose que j'appelle le paradoxe des mouvements sociaux. C'est-à-dire que sous l'affirmation de "c'est un gouvernement des mouvements sociaux», en réalité s'est consolidé un État qui désarme les mouvements sociaux et où se construit un ensemble d'organismes sociaux, de corporations sociales, pour établir un programme de gouvernement décidé depuis «en-haut » et qui annule à nouveau toute possibilité de participation. Comment cela a-t-il été possible ? Je crois que c'est grâce à la conservation de la structure argumentative. C'est ici que l'apport de la théorie critique est très intéressant. Parce que la théorie critique, certaines approches de l'École de Francfort - et ici beaucoup de collègues la cultivent de manière très fine - avaient insisté sur le fait que dans le terme lutte de classes, il fallait prioriser la lutte avant de prioriser ou d'essayer de comprendre le Sujet. Autrement dit, il fallait voir le déploiement de l'antagonisme avant de voir et de prendre ces sujets comme un objet d'étude de l'analyse. Je crois qu'il y a là une piste intéressante, justement pour rompre avec le paradoxe dont je parlais : bien que nous ayons vécu des gouvernements ouvriers, ils ont été un désastre et ont armé le capitalisme d’État réellement existant pendant de nombreuses années.

Depuis, on a pu voir également ce qu'a donné la théorie des mouvements sociaux : elle a permis la consolidation de gouvernements progressistes qui parlent de gouvernements de mouvements sociaux, mais qui sont en fait d'un point de vue structurel exactement calqués sur les précédents. Je sens pour ma part qu'il est plus intéressant d'une part de mettre l'accent sur la lutte, c'est-à-dire sur le déploiement concret, systématique, quotidien de l'antagonisme, soit sur le registre de ce qui se passe réellement, et d'autre part de façon conjointe, de discerner ce que disent vouloir ceux qui luttent, ce qu'ils vont faire et ce qu'ils ne disent pas, ne parviennent pas à exprimer, ce qu'ils expriment, mais ne font pas. C'est-à-dire, aller voir avec beaucoup de détails ce qui se passe dans la lutte pour pouvoir comprendre - c'est un peu l'idée que je développe systématiquement - ce déploiement de l'antagonisme dans ses portées concrètes (à savoir des blocages généralisés, des mobilisations et où, qu'est-ce qu'elles parviennent à arrêter et où), tout en essayant de chercher l'horizon intérieur. J'appelle horizon intérieur, un peu dans la lignée de Bloch, cet ensemble de choses contradictoires : ce qu'ils disent dans les tracts, ce qu'ils disent dans les annonces, la façon dont ils argumentent quand ils négocient, mais aussi ce qu'ils font et ne disent pas, ce qu'ils disent et ne font pas, ce qui peut s'exprimer dans des termes plus intimes, mais ne parvient pas à être exprimé publiquement, pour essayer de comprendre finalement un horizon de désir 
qui se déplace et ne s'éteint pas. C’est, je crois, ce que j’ai essayé de promouvoir toutes ces années, et que maintenant - pour revenir à la question antérieure — je veux aussi essayer de faire en termes académiques et théoriques, à propos de cette insubordination, à partir de cette vague de mouvements de femmes rénovés, en recommençant à le pratiquer. C'est-à-dire, pouvoir aussi avoir une réflexion à la première personne, ce qui est quelque chose de très important.

Donc, le concept de classe sociale ne disparaît pas, il est secondaire à celui de lutte et dans cette optique, les collègues travaillent beaucoup la question de la subjectivité. Ici, ceux qui cultivent la théorie critique ont nommé leur séminaire subjectivité et théorie critique. Il se focalise sur la manière dont vont apparaître et se déployer ces processus de subjectivation. Pour ma part, c'est là où je commence à mettre de la distance avec eux : il me semble que ces processus de subjectivation, il faut les chercher dans leur incarnation dans le quotidien. C'est pourquoi je me suis focalisée à cette période sur un domaine de recherche appelé réseaux communautaires. Comment dans de multiples endroits, ces liens que l'on peut appeler communautaires, se régénèrent-ils ? Premier problème : que va-t-on appeler liens communautaires ? Comment va-t-on comprendre la production du commun? Quels types de relations s'expriment là ? Ce sont des liens que l'on va rencontrer dans plusieurs endroits, avec des similitudes. Question suivante: pourrions-nous déjà commencer à penser à quelqu'un qui paraît être là chaque fois que se déploie une lutte et se réélabore une espèce de corps social mobilisé, sans être exactement un mouvement social. Je ne veux pas me figer dans ce corps social, je veux me concentrer sur la dynamique de la forme du lien et sur la nouveauté qui s'y produit. Je tiens également à me focaliser sur les choses qui corrompent ces liens dans les moments non-extraordinaires, dans les autres moments que ceux de la lutte déployée, donc dans les moments quotidiens : comment ces liens se corrompent, comment ils commencent à se diluer ou se conservent et vont être soignés.

Le projet de recherche visait donc à poser de nouveau un intérêt pour un sujet collectif qui ne soit pas considéré comme donné au préalable, mais un sujet collectif dont on étudie la production et l'auto-production de manière très systématique dans le quotidien. Et là, le plus important, en récupérant de nouveau l'approche féministe, dans ce cas celle de Federici, c'est que nous avons pensé mettre au centre les différents processus impériaux, symboliques et (in) tangibles de reproduction de la vie collective, en incluant tout le champ d'action, parce que la reproduction de vie sociale n'est pas uniquement liée au processus de procréation. Le fait de mettre la production de la vie quotidienne comme condition centrale de ce capitalisme dans lequel nous sommes, c'est recommencer à rendre visible le problème, non pas de la subjectivité et de la subjectivation - qui est important, mais qui n'est pas le seul pertinent -, mais de la capacité de se produire soi-même comme sujet de transformation de l'existant, susceptible de politisation. De là, le nom un peu bizarre du domaine de recherche dans lequel je m'inscris : entrelacement communautaire et formes du politique. Voici certaines des ques- 
tions qui sont posées : quelles sont les nouveautés politiques ? Quelles choses non totalement libérales vont se réinstaller? Quelles autres formes de production d'accords vont être cultivées de manière plus intensive ? Là s'esquisse ce que l'on peut connaître et comprendre des pratiques indigènes qui s'amplifient et sont réactualisées, parce que ce n'est pas seulement un héritage et ce n'est pas qu'une répétition : les pratiques indigènes sont toujours une réactualisation présente d'éléments dont l'origine est connue par ceux qui sont dans ces réseaux. Il s'agit donc de saisir cette dynamique, mais aussi les choses qui sont en train d'apparaître. Par exemple, il y a un ensemble de pratiques intéressantes de solidarité, d'aide mutuelle, etc. qui commencent à se développer parmi les gens de ta génération, qui a du mal avec le travail, à parvenir à cette stabilité minimale que j'ai évoquée au début de cet entretien. Des pratiques communautaires commencent à être déployées et à être expérimentées. Mais on manque d'éléments pour comprendre ce phénomène, au-delà de ce qu'il représente comme forme collective de survie. La question théorique et politique est donc: comment pouvons-nous comprendre cette situation à laquelle se confronte la jeune génération dans le Sud global ? Et comment d'autres traditions peuvent nourrir ce qu'aujourd'hui, un ensemble de jeunes urbains bien formés sont en train de faire et qu'ils ne parviennent pas à réaliser, bien que très connectées, parce qu'ils sont pris par l'exigence de trouver les moyens de survivre de façon individuelle. Ce qui est précisément ce qu'ils sont en train de vivre, même s'ils volent en avion ou voyagent dans divers endroits. Ils sont réellement perdus, pour le dire avec des mots clairs, du moins c'est ce que moi je vois. Je ne connais pas le cas de la Belgique, mais avec certains groupes d'étudiants et camarades nordaméricains, ce qu'ils vivent est une barbarie : ils ne savent pas ce qu'ils vont faire le mois qui vient, ils doivent sans cesse avoir de nouveaux projets. Leur vie est rompue. Les camarades espagnols vivent la même chose. Comment aborder ce problème, comment réussir à imaginer, ou quels éléments pourraient contribuer à imaginer des pistes de réflexion pour penser une certaine stabilité ? C'est un problème politique.

Aujourd'hui, le concept de commun rencontre un grand succès, tant dans les luttes que dans la recherche. Comment l'expliquez-vous? Peut-on considérer ce concept comme un concept global?

RGA : Je crois que ce concept du commun a à voir avec la manière dont se garantit ultimement la possibilité de soutenabilité de la vie en situation d'offensive totale du capital. Il n'est pas étonnant, dans ce sens, que les tissus communautaires les plus denses soient les tissus communautaires indigènes en Amérique Latine, parce que ce sont eux plus que les autres qui ont vécu le drame de la conquête. En effet, ce sont les populations indigènes qui ont expérimenté la chute brutale de tout leur monde. Elles se sont appropriées ou ont essayé de récupérer et de déformer les institutions coloniales de contrôle et d'organisation de la population qu'imposait l'Empire Espagnol. En les dénaturalisant, en les bouleversant, en les perturbant, etc. elles ont réussi à conserver un 
ensemble de biens matériels en total accord avec des pratiques qui avaient été générées pour prendre soin et fructifier ces biens matériels : l'eau, les bois, les terres, les semences, les pratiques culturelles... Ce qui a été historiquement soigné par ces populations et a constitué la base-même de la possibilité de leur existence et de sa poursuite constitue un lien central avec cette offensive atroce que nous vivons partout depuis presque quarante ans.

Cette offensive est possible parce que les limites au capitalisme que la génération de tes grands-parents et de mes parents ont réussi à établir, tombent ou sont attaquées. Cette génération, en se battant durement, a réussi à inscrire au niveau de l'État un ensemble de droits sociaux qui commencent à être démantelés aujourd'hui. Et cela met tout le monde, littéralement, en grande difficulté. Cet ensemble d'expropriation à grande échelle s'apparente d'un côté à une sorte de processus de dépossession à la Harvey. Il s'agit là non seulement des biens communs en tant que tels, mais aussi des biens communs qui ne sont pas naturels, qui existent en tant qu'ils sont sociaux, qu'ils ont été soignés, conservés, fructifiés sous des modèles distincts de celui du capital, et qui ont été spoliés. Cette grande vague de dépossession commence là, mais ne se termine pas là : cette dépossession est aussi celle des capacités politiques, des ruptures des liens, de cette barbarie de la guerre contre les femmes, de toutes ces tâches de reproduction qui se retournent maintenant contre les femmes isolées sous prétexte de les émanciper des foyers (je me réfère au soin des enfants, des vieux,...), de manière invisible et privée. Il s'agit aussi ce vol brutal de temps dans les milieux urbains. Tout cela s'est fait ressentir de façon globale, surtout durant la première décade de ce siècle, quand s'est écroulée lieu après lieu l'illusion que le marché allait être la solution à tout. Mais la question de l'État ou la stratégie de la génération antérieure d'inscrire tous les droits dans l'État est encore à fleur de peau. En réalité, cette stratégie a crée une forme étrange du privé parce que l'État, dans cette acceptation du public, est toujours resté médiatisé par certains qui monopolisaient la possibilité de décider de la voie à suivre. Donc ce n'était pas non plus perçu comme une option d'en bas. Cela explique l'idée de parler du commun, qui est apparue en de nombreux endroits.

Ici, nous dialoguons avec des gens qui réfléchissent à cela depuis de nombreuses années, comme Silvia Federici évidemment, mais aussi avec toutes les personnes de la revue The Commoners, qui mènent cette recherche très importante sur les commons (ici en anglais, et au pluriel). Nous cherchons à leur montrer que ce qui est important et qui déplace la compréhension des communs comme étant des biens chosifiés, c'est de considérer le commun comme une relation sociale, de la même façon que le capital n'est pas une somme d'argent, mais une relation sociale. Marx nous l'a dit, et l'a très bien expliqué au XIXe siècle. Nous essayons de penser de façon systématique le commun et la production du commun, à la fois comme concrétisation d'une richesse matérielle qui est soignée, se conserve et se fructifie, mais aussi comme une relation sociale. Certaines particularités de cette relation sociale ont été mises en évidence par la lutte, et c'est 
aussi ce que nous tentons de comprendre. Il s'agit par exemple de la centralité de l'assemblée, du fait que tout le monde doit être inclus dans la production et la co-production de la décision politique, qu'il faut penser des formes distinctes d'autorité qui ne sont pas de type hiérarchique (la hiérarchie qu'impose le capital). Tout cela est directement lié avec des éléments documentés dans un autre langage dans le mouvement indigène, comme ce désir de construire de nouvelles formes d'auto-gouvernement dans les territoires localisés. Donc, le point essentiel de notre proposition, que nous cultivons dans cet espace de recherche, c'est de penser le commun comme une relation sociale. On se consacre à regarder avec beaucoup d'attention quels sont les traits généralisables qu'on peut trouver en voyant les différents processus de production du commun. C'est dans ce sens que va notre réflexion, et nous avons une série de choses publiées là-dessus.

Comment faire dialoguer les luttes et les recherches en Amérique Latine et en Europe, dans le Sud global et le Nord global?

RGA : Moi je l'ai vécu comme quelque chose de très compliqué. Je remarque deux choses avec les camarades européens : d'un côté, les processus d'individualisation sont beaucoup plus marqués dans la structuration de votre subjectivité que dans la nôtre. Autrement dit, en Amérique Latine, c'est encore très commun de rencontrer des familles très élargies. Je ne suis pas en train de dire que la famille est moins valorisée en Europe, mais finalement que le rapport à la famille va susciter des processus de subjectivation distincts, qui prépare davantage à l'organisation et délègue des capacités pour se penser de façon plus collective, même si c'est aussi parfois de façon oppressive, ce qui est un très gros problème. Au final, cela centre beaucoup plus l'analyse sur la question de l'interdépendance, qui est la question centrale de la production du commun. Ce processus violent d'individualisation et de désir d'autosuffisance dans lequel vous vous êtes sociabilisés, les hommes et femmes qui sont nés en Europe ou dans le Nord global, n'est pas encore abouti ici. Vous avez appris que la relation marchande est la relation essentielle et parfois qu'il s'agit de la seule imaginable. La notion d'autosuffisance qui fonde l'individualisme est alors difficilement critiquable, et il est compliqué de penser des liens d'interdépendance.

À côté de ça, il y a autre chose que je perçois parfois comme complètement intériorisé en vous, et qui, à moi du moins, me donne beaucoup de difficultés pour dialoguer avec des publics européens. Il s'agit de comprendre la critique que la politique des droits est une politique qui amène finalement à une marchandisation de prérogatives qui s'inscrivent parfaitement dans l'ordre capitaliste. Habituellement, j'ai beaucoup de mal à travailler cela, à le mettre en discussion avec des collègues européens parce que ce sont leurs générations passées qui ont lutté et ont réussi à inscrire des droits. En parlant de générations passées, je parle des travailleurs, pas des colonisateurs uniquement, autrement dit pas le Nord nord global ou l'élite globale. Ces droits inscrits ont débouché sur 
une forme étatique dans laquelle ils ont placé tous leurs pions, et c'est ce qu'ils ont transmis comme connaissance. Mais c'est votre histoire, ce n'est pas l'histoire de tout le monde. C'est très laborieux par moments d'arriver à le penser pour vous, et je rencontre une grande difficulté à le communiquer.

Maintenant, je vois aussi une génération, la tienne, c'est-à-dire les jeunes qui ont entre 20 et 35 ans, qui a été convertie en indigènes. Vous êtes les nouveaux indigènes ! Seuls certains d'entre vous vont parvenir à s'intégrer, au prix de renoncer à euxmêmes. Nous commençons à voir, à différents niveaux, une espèce de rébellion de la jeunesse qui est en recherche d'autre chose. Je crois que c'est très important de pouvoir discuter de cela, et trouver des termes pour un dialogue dans des conditions équilibrées de respect et d'égalité, où les collègues européens sont aussi disposés à mettre en doute des parties fondatrices de la structure de leurs propres appareils psychique et de significations. Il s'agit de prendre au sérieux ce que nous pouvons leur dire d'ici, à savoir qu'il y a des problèmes qui n'ont pas de solution. Et nous savons qu'ils n'en ont pas parce que nous ne sommes pas restés tant à l'intérieur du capitalisme. En plus, presque n'importe quel Latino-Américain lettré qui a fait des études universitaires a une connaissance de l'histoire européenne absolument plus grande que celle que vous avez sur le reste du monde, de la même façon que le féminisme nous apprend que le dominé sait beaucoup plus que le dominant parce qu'il connaît ce qu'il pense et il connaît les faiblesses du dominant. Reconnaître cela serait aussi un élément qui serait en jeu à l'heure d'ouvrir des dialogues. Il serait très important de réussir aujourd'hui, dans une espèce de dialogue séculier, à éclairer les angles morts de chacun, parce qu'il y a des choses que nous ne savons pas, mais que vous ne nous dites pas, parce qu'elles ne paraissent pas être principales. Et vice-versa. Mais depuis la crise de 2008, je sens aussi que cela change fortement et ça me réjouit beaucoup. Et nous sommes en train d'essayer de commencer des discussions plus fortes, pour commencer avec ceux avec qui nous pouvons dialoguer plus facilement, c'est-à-dire ceux qui parlent notre langue, les Espagnols et en partie les Italiens. Avec les autres, c'est encore pour le moment plus difficile. 